\title{
Positioning Analysis of Multiple Antennas in a Dense RFID Reader Environment
}

\author{
Kin Seong Leong, Mun Leng Ng, Member, IEEE, Peter H. Cole \\ Auto-ID Laboratory, School of Electrical and Electronic Engineering, the University of Adelaide \\ \{kleong,mng,cole\}@eleceng.adelaide.edu.au
}

\begin{abstract}
The study and analysis of RFID antenna positioning is important for supply chain large-scale deployment. In a dense reader environment, there will be multiples of RFID readers and readers interference will reduce the reliability and efficiency of the RFID system. In the worst case, the interference would paralyse an entire RFID system. This paper explores antenna positioning in an RFID deployment zone and aims to provide guidelines on safe distance between antennas in a dense reader environment or even in a "Listen Before Talk" regulated area. By planning of antenna positioning, a RFID system can be optimised and its interference to other $R F$ systems in the surroundings can be minimised. All the simulations results presented in this paper are obtained using MATLAB.
\end{abstract}

\section{Introduction}

Radio Frequency Identification (RFID) has recently received much attention from various parties involved in supply chain management. It is believed that RFID can bring revolutionary changes to supply chain, replacing the existing barcode systems. Some prominent companies and organizations have already shown interest in RFID. For example, Wal-Mart, one of the biggest retailers in the USA, has mandated to its top 100 suppliers the use of RFID in its supply chain by Jan 2005 [1]. Also, the USA Department of Defense has mandated the use of passive RFID by Jan 2005 in its supply chain [2].

One of the biggest challenges in RFID large-scale deployment is the RFID signal interference problem. The problem is not only limited to RFID devices disrupting the operation of other RFID devices operating in close proximity, but also includes RFID devices disrupting the operation of other RF devices in nearby frequency bands. Regulatory bodies have set some strict restrictions on RFID radiation to minimize the RFID interference problem. The concept of "Listen
Before Talk" provision has been included in the latest European regulation, ETSI EN 302208 [3], which put severe restrictions on RFID deployment. The main purpose of this paper is to investigate the art of antenna positioning to optimize RFID field performance, while adhering to strict regulations. Intensive simulations were carried out to estimate RFID reader signal strength at different distances away from the transmitting antenna, either operating in a same channel or in any neighbouring channels. It is hoped that this paper can provide sufficient and useful guidelines for the development of RFID systems with optimized performance.

In the next section, some relevant theories that form the core of the simulation software written to analyse antennas positioning will be explained, along with some of the closely related regulations. The third section explains the concepts behind the simulation software developed in a MATLAB environment. The fourth section includes both the simulation results and discussions of various antenna positionings, and the final section presents a conclusion for the work done and materials presented in this paper.

\section{Background}

In this section, the discussions are focused on the concept of path loss and the path loss model chosen for implementation in the simulation software. A more extensive explanation on the path loss model can be found in [4]. Also included in this section is the antenna gain pattern used and also some regulations and standards applicable to RFID systems.

\subsection{In-building path loss}

The power transfer ratio for a pair of lossless antennas in free space with optimum orientation is given by: 


$$
\frac{\mathrm{P}_{\mathrm{r}}}{\mathrm{P}_{\mathrm{t}}}=\mathrm{g}_{\mathrm{t}} \mathrm{g}_{\mathrm{r}}\left(\frac{\lambda}{4 \pi \mathrm{d}}\right)^{2}
$$

where $\lambda=$ wavelength; $P_{t}=$ transmitted power; $P_{r}=$ received power; $g_{t}=$ transmitter antenna gain; $g_{r}=$ receiver antenna gain; $d=$ separation distance between antennas. The factor $\left(\frac{\lambda}{4 \pi \mathrm{d}}\right)^{2}$ in (1) is separated from the effects of the transmitter and receiver antenna gain and will be referred as the free space path loss.

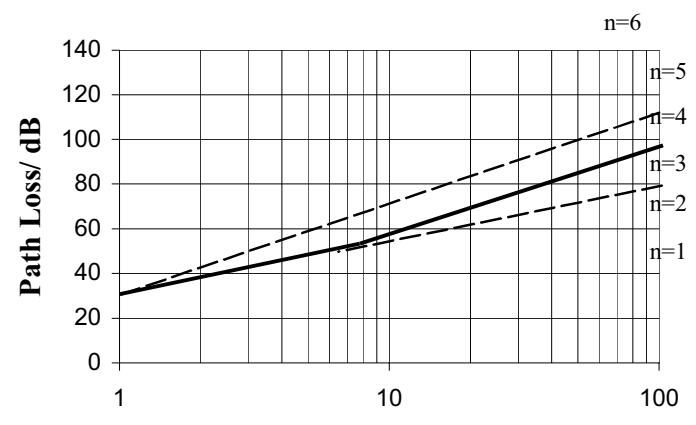

Transmitter-Receiver Seperation/ $\mathbf{m}$

\section{Figure 1. Plot of in-building path loss model against distance [4]}

An in-building path loss is a path loss that occurs in a physical building, and take in consideration of path obstruction, reflection, absorption and other attenuation effects introduced by the presence of objects inside a building. The in-building path loss model chosen for the purpose of simulation is given in (2), and is explained by Rappaport [5]:

$$
\operatorname{PL}(\mathrm{dB})=\operatorname{PL}\left(d_{0}\right)+10 n \log \left(\frac{d}{d_{0}}\right)
$$

where $\quad d_{0}=$ arbitrary reference distance; $n=$ a value that depends on the surroundings and building types; $d$ $=$ the separation distance between two antennas. $d_{0}$ will be chosen as $1 \mathrm{~m}$ and $\operatorname{PL}\left(d_{0}\right)$, which is the in-building path loss at $1 \mathrm{~m}$ away, will be approximately the same as the free space path loss at $1 \mathrm{~m}$ away, as room reflections are not huge at this small distance. As explained in (2), $\mathrm{n}$ is an empirical value that depends on the surroundings and building types, and is only obtainable through experiment. An environment with high $\mathrm{n}$ is a hostile environment for radiation, and its inbuilding path loss will be higher compared to the case in low $\mathrm{n}$ environment. For the purpose of simulation, the in-building path loss model from (2) was modified slightly and is given in (3) and is plotted as shown in Fig. 1. This model is discussed in greater detail in a similar study on RFID reader collision problem [4].

$$
P L(d B)=\left\{\begin{array}{lc}
32+25 \log \left(\frac{d}{1}\right) & 0 \leq d<8 m \\
23+35 \log \left(\frac{d}{1}\right) & d \geq 8 m
\end{array}\right.
$$

\section{2. "Listen Before Talk"}

The concept of "Listen Before Talk" provision is introduced in the European regulation as outlined in ETSI EN 302 208-1 V1.1.1 (2004-09) [3]. A reader must "listen" and confirm that a particular channel is unoccupied before it can use that particular channel to interrogate any tag. The transmit power and the corresponding threshold values are extracted from [3] and are presented in Table 1, all in terms of Effective Radiated Power (ERP).

Table 1: Transmit and threshold power

\begin{tabular}{|c|c|c|}
\hline ERP (W) & ERP (dBW) & Threshold (dBW) \\
\hline Up to 0.1 & Up to -10 & $\leq-113$ \\
\hline 0.1 to 0.5 & -10 to -3 & $\leq-120$ \\
\hline 0.5 to 2.0 & -3 to 3 & $\leq-126$ \\
\hline
\end{tabular}

\subsection{An RFID directional antenna}

As shown by (1), antenna gain must be taken into consideration, apart from path loss, to relate transmitted power to received power. A typical RFID antenna is a directional circularly polarised antenna with a gain of $6 \mathrm{dBi}$. The polar plot of the gain of a typical RFID antenna is shown in Fig. 2 below, and has been used to simulate and analyse the antenna positioning in dense reader environment presented in this paper.

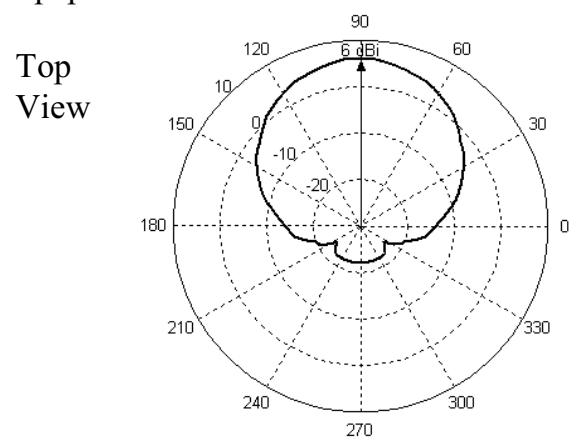

Figure 2. Polar plot of the antenna gain

\subsection{Frequency spectrum channelling for RFID}

One of the biggest challenges in frequency spectrum channelling is the sideband interference, where the sideband of a particular channel interferes with the transmission in a neighbouring channel. Hence, under the latest UHF Class 1 Generation 2 protocol [6], the sidebands of a transmission must adhere to the limits 
as shown in Fig. 3. The $\mathrm{dBch}$ is defined as decibels referenced to the integrated power in the reference channel. The simulation software developed uses this limit to investigate interactions of readers operating using different frequency channels.

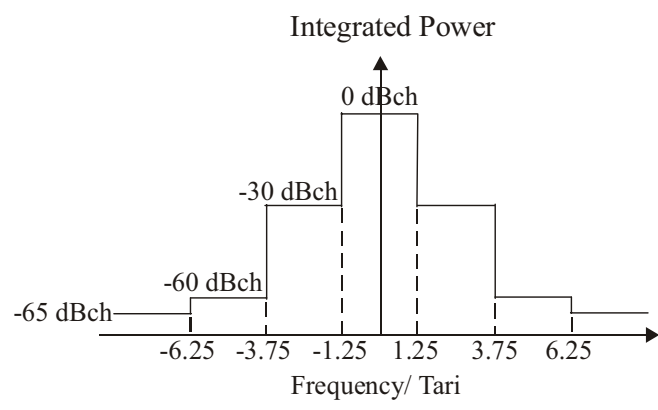

Figure 3. Transmit mask for denseinterrogator environments [3]

\section{Simulation concepts}

The simulation script is written in MATLAB. The resolution of the simulation results on antenna signal strength can be adjusted, but by default the resolution is $1 \mathrm{~m} \times 1 \mathrm{~m}$. Another resolution used is $10 \mathrm{~m} \times 10 \mathrm{~m}$. Antennas used in the simulation are directional circularly polarised antenna, with antenna gain shown in Fig. 2, and is located $1 \mathrm{~m}$ above ground level (Refer Fig. 6 for visualization of antenna configuration). By specifying a transmit power, (1) is used to compute the received power with the gain specified in Fig. 2, and path loss is computed from (3). The simulation result is in graphical form and is able to show the received power at any location, bounded by a predefined limit, when a receiving antenna of $0 \mathrm{~dB}$ gain is used, and is from the top view at $1 \mathrm{~m}$ above the ground.

\section{Simulation results and discussions}

\subsection{Simulation antenna positioning}

The antenna simulation covers a larger area to identify available zone to deploy a second antenna working in a same channel. From Table 1, the minimum threshold is $-126 \mathrm{dBW}$ for transmission of 2W ERP. The simulation result as shown in Fig. 4, and shows that for the next antenna to be able to operate in the same channel with the initial antenna under consideration, both antenna must be separated by a distance of around $350 \mathrm{~m}$ away in the horizontal direction. If the second antenna is to be placed in a back to back position with the first antenna, the minimum distance between them would be shorter, which is slightly more than $200 \mathrm{~m}$.

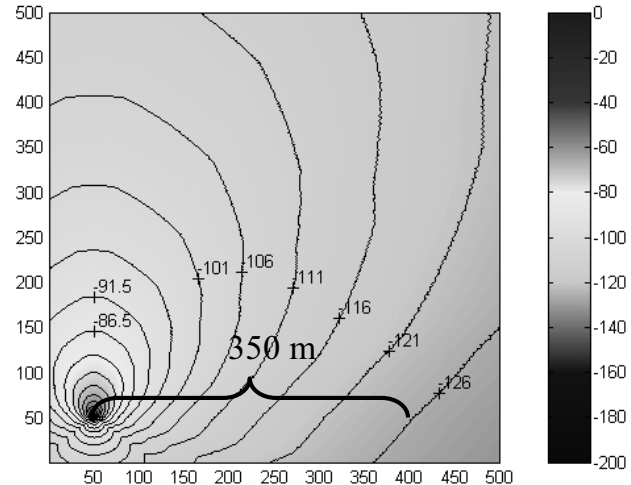

Figure 4. Simulation results (axes in $\mathrm{m}$, received power in $\mathrm{dBW}$ )

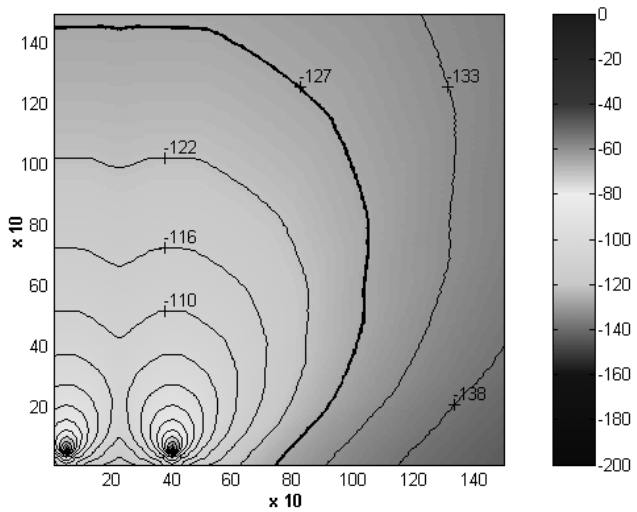

Figure 5. Simulation results (axes in $10 \times \mathrm{m}$, received power in $\mathrm{dBW}$ )

A second antenna is deployed $350 \mathrm{~m}$ horizontally away from the first one, the result is as shown in Fig. 5. The bold line is the approximate safe boundary of the deployment of the third reader operating in a same channel. The distance is approaching $1.4 \mathrm{~km}$ in front of both antennas and $400 \mathrm{~m}$ horizontally. This shows that for antennas transmitting in a same frequency, they have to be at a great distance apart.

\subsection{One antenna on the ground}

Another configuration is to place an antenna on the ground with the interrogation field projected upwards, as shown in Fig. 6. It is assumed that an antenna located $2 \mathrm{~m}$ above the ground with the interrogation field projected downwards (in Fig. 6) will have a similar radiation power at far field as compared with the antenna on the ground. By replacing the rightmost antenna in Fig. 5 with an antenna on the ground, it is discovered that the boundary to deploy another reader operating in the same frequency channel moves closer to the first and second antennas. 


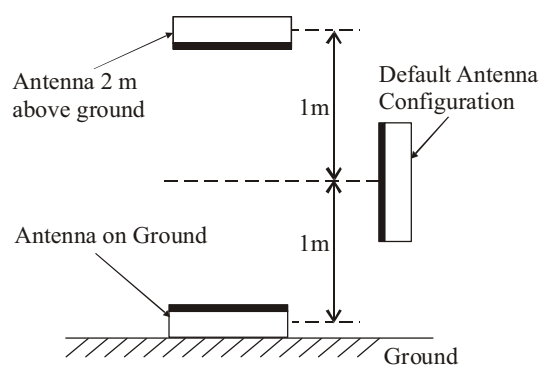

Figure 6. Configuration of antenna on ground with respect to normal configuration

\subsection{Antennas operating at different channels}

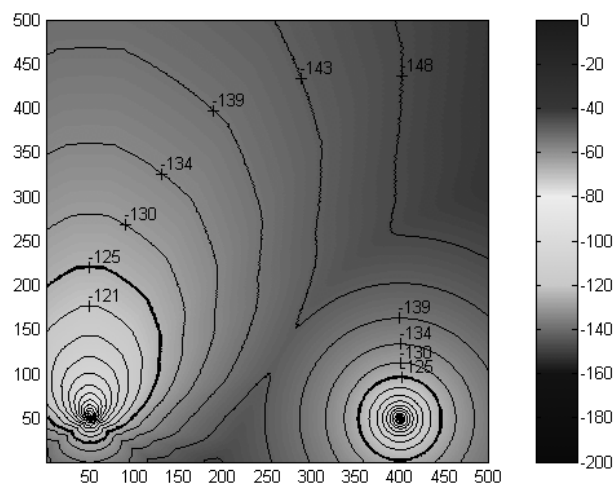

Figure 7. Simulation results (axes in $\mathrm{m}$, received power in $\mathrm{dBW}$ )

In this section, the effect of neighbouring channels will be investigated. The limit of sideband interference applied is as shown in Fig. 3. For two readers operating in direct neighbouring channels, the simulation results on the frequency band of interest are as shown in Fig. 7. This result shows that for a transmission operation in the band of interest, the antenna must be placed $50 \mathrm{~m}$ away in all direction from an antenna on the ground. For an antenna on default configuration (Refer Fig. 6) operating at direct neighbouring channel, the distance is around $180 \mathrm{~m}$ from the front, $45 \mathrm{~m}$ from both sides and $30 \mathrm{~m}$ from the back. Table 2 shows all the simulation results corresponding to different antenna configurations. From the table, it is very difficult to conclude which configuration is better than the other. It all depends on the type of applications a RFID system is deployed for. However, this table is sufficient to serve as a guideline on the safe distance between antennas in a deployment zone. The only restriction is that all the readers must be able to be pre-programmed to operate only in a pre-selected frequency channel.
Table 2. Safe distance for different antenna configurations

\begin{tabular}{|c|c|c|c|c|}
\hline \multirow{2}{*}{ Channel } & \multicolumn{2}{|c|}{ Antenna on Default Configuration } & \multirow{2}{*}{ Antenna on } \\
\cline { 2 - 4 } & Front $(\mathrm{m})$ & Side $(\mathrm{m})$ & Back $(\mathrm{m})$ & \\
\hline 0 & 1400 & 350 & 210 & 320 \\
\hline 1 & 180 & 45 & 30 & 50 \\
\hline 2 & 130 & 25 & 15 & 35 \\
\hline 3 & 95 & 20 & 10 & 30 \\
\hline
\end{tabular}

\section{Conclusion}

This paper has presented a path loss model that can be applied to predict the signal strength of RFID interrogation signal at a certain distance away from a transmitting antenna. Simulations results are presented, together with some insights and discussions. Safe distances between antennas are suggested and these results can be used in RFID large-scale deployment area to avoid readers interference and to adhere to strict regulations, such as the European "Listen Before Talk" provision. It has been shown that strict regulations will in fact limit large-scale deployment of RFID system. By using readers synchronization and frequency channelling, the situation can be improved. Actual field testing will be carried out in the future, especially in warehouses, where dense RFID reader environments may most likely exist.

\section{References}

[1]D. Barlas, "Wal-Mart's RFID mandate," 4 June 2003. [Online]. Available: http://www.line56.com/articles/ default.asp?ArticleID=4710.

[2] Department of Defence United States of America, "Radio frequency identification (RFID)," 3 May 2005. [Online]. Available: http://www.acq.osd.mil/log/rfid/ index.htm.

[3] ETSI EN 302 208-1 V1.1.1 (2004-09), [Online] www.etsi.org.

[4] K. S. Leong, M. L. Ng, P. H. Cole, "The reader collision problem in RFID systems", presented in MAPE 2005, Beijing, August, 2005.

[5] T. S Rappaport, Wireless Communications - Principles and Practice, Prentice Hall, Second Edition, 2002.

[6] EPCglobal, "EPC radio-frequency identity protocols class-1 generation-2 UHF RFID protocol for communications at $860 \mathrm{MHz}-960 \mathrm{MHz}$ version 1.0.9," EPCglobal Standard Specification, 2004. 